\title{
LHC bounds on large extra dimensions
}

\section{Roberto Franceschini, ${ }^{a}$ Pier Paolo Giardino, ${ }^{b}$ Gian F. Giudice, ${ }^{c}$ Paolo Lodone, ${ }^{d}$ and Alessandro Strumia ${ }^{b, e}$}

${ }^{a}$ Institut de Théorie des Phénomènes Physiques, EPFL, CH-1015 Lausanne, Switzerland

${ }^{b}$ Dipartimento di Fisica dell'Università di Pisa and INFN, Largo Bruno Pontecorvo 3, 56127 Pisa, Italy

${ }^{c}$ CERN, Theory Division, CH-1211, Geneva 23, Switzerland

${ }^{d}$ Scuola Normale Superiore and INFN,

Piazza dei Cavalieri 7, 56126 Pisa, Italy

${ }^{e} N I C P B$

Ravala 10, 10143 Tallinn, Estonia

E-mail: roberto.franceschini@epfl.ch, pierpaolo.giardino@pi.infn.it, gian.giudice@cern.ch, p.lodone@sns.it,

Alessandro.Strumia@mail.df .unipi.it

ABSTRACT: We derive new dominant bounds on the coefficient of the effective operator generated by tree-level graviton exchange in large extra dimensions from $p p \rightarrow j j$ data at LHC: $M_{T}>2.1 \mathrm{TeV}$ (ATLAS after $3.1 / \mathrm{pb}$ of integrated luminosity), $M_{T}>3.4 \mathrm{TeV}$ (CMS after $36 / \mathrm{pb}$ ), $M_{T}>3.2 \mathrm{TeV}$ (ATLAS after $36 / \mathrm{pb}$ ). We clarify the role of on-shell graviton exchange and compare the full graviton amplitude to data, setting bounds on the fundamental quantum-gravity scale.

KEYWORDS: Phenomenology of Large extra dimensions

ARXIV EPRINT: 1101.4919 


\section{Contents}

1 Introduction 1

2 Fit to the graviton-exchange effective operator 3

3 Tree-level graviton exchange $\quad 7$

$3.1 \delta=1 \quad 9$

$\begin{array}{lll}3.2 & \delta>1 & 11\end{array}$

$4 \quad$ Fit to the full graviton-exchange amplitude 12

$\begin{array}{llr}5 & \text { Conclusion } & 14\end{array}$

\section{Introduction}

With the start of the LHC program, experiments are already testing directly some of the theoretical ideas about new physics at the electroweak scale. In one popular scenario, which will be considered in this paper, Standard Model fields are confined on a 3-dimensional brane, while gravity propagates in the full $D$-dimensional space, with $\delta$ flat and compactified extra spatial dimensions $(D=4+\delta)[1]$. This scenario allows for quantum gravity at the weak scale and could therefore be a solution to the Higgs mass hierarchy problem. Even without knowledge of the exact model for quantum gravity at the weak scale, we can make some definite predictions for collider experiments using either low-energy effective theory or semi-classical approximation, which can provide valid descriptions in certain kinematical domains. When experimental data are compared with expectations, it is then important to assess the validity of the approximations used in the theoretical calculations.

We can identify five different kinds of LHC signals which allow for a theoretical interpretation in terms of $D$-dimensional gravity.

1. Missing $p_{T}$ from emission of massive gravitons constituting the Kaluza-Klein tower. This signal is within control of the low-energy effective theory as long as the graviton energy is less than an ultraviolet cutoff $\Lambda_{\text {eff }}$, which characterizes the onset of the new quantum-gravity theory. Validity of the perturbative expansion sets an upper bound on the cutoff

$$
\Lambda_{\text {eff }}<[\Gamma(2+\delta / 2)]^{\frac{1}{2+\delta}}(4 \pi)^{\frac{4+\delta}{4+2 \delta}} M_{D}
$$

where $M_{D}$ is the $D$-dimensional Planck mass in the notation of [2]. This upper bound is saturated only when gravitons become fully strongly-interacting before entering the new regime of the underlying theory, and thus $\Lambda_{\text {eff }}$ could actually turn out to be much smaller. This does not mean that missing $p_{T}$ signals above $\Lambda_{\text {eff }}$ vanish, but simply that they are not calculable without knowledge of the full theory. 

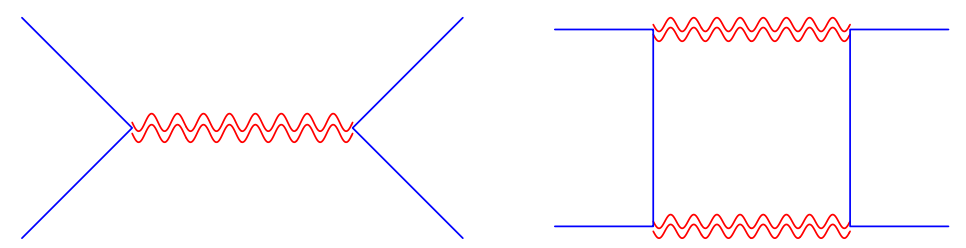

Figure 1. Figure 1a: Tree-level graviton exchange generating the dimension-8 operator $\mathcal{T}$. Figure 1b: One-loop graviton exchange generating the dimension- 6 operator $\Upsilon$.

2. Tree-level exchange of gravitons (figure 1a) generating the effective dimension-8 operator $\mathcal{T}[2-4]$

$$
\mathcal{L}_{\text {int }}=c_{\mathcal{T}} \times \mathcal{T}=\frac{8}{M_{T}^{4}} \times \frac{1}{2}\left(T_{\mu \nu} T^{\mu \nu}-\frac{T_{\mu}^{\mu} T_{\nu}^{\nu}}{\delta+2}\right)
$$

where $T_{\mu \nu}$ is the SM energy-momentum tensor. As discussed in section 3, in most cases the dominant contribution to this operator comes from the ultraviolet end of the graviton spectrum. Therefore the parameter $M_{T}$ cannot be computed without knowledge of the underlying quantum-gravity theory. The case $\delta=1$ (and, to a certain extent, $\delta=2$ ) provides an interesting exception.

3. Virtual graviton exchanges at one-loop level (figure 1b) can become more important than tree-level effects because they induce dimension- 6 effective operators, as opposed to the dimension- $8 \mathcal{T}$ operator [5]. For pure graviton virtual intermediate states, a unique dimension- 6 operator is generated

$$
\mathcal{L}=c_{\Upsilon} \times \Upsilon, \quad \Upsilon=\frac{1}{2}\left(\sum_{f} \bar{f} \gamma_{\mu} \gamma_{5} f\right)\left(\sum_{f} \bar{f} \gamma^{\mu} \gamma_{5} f\right)
$$

where $f$ is any SM quark or lepton. As in the case of tree-level graviton exchange, the coefficient $c_{\Upsilon}$ is fully sensitive to the ultraviolet completion of the theory and can be related to the fundamental parameters $M_{D}$ and $\delta$ only by specifying a cutoff procedure.

4. Dijet events at large invariant mass and large rapidity separation. In this kinematic regime, gravitational scattering can be reliably computed in the eikonal approximation [6]. This is because scattering processes at center-of-mass energy larger than $M_{D}$ (the so-called transplanckian region) are governed by classical dynamics and any quantum-gravity effect is subdominant.

5. Black holes. Black-hole formation and decay is expected to occur in the transplanckian region when the impact parameter becomes smaller than the corresponding Schwarzschild radius [22-24]. Therefore it supplants gravitational scattering, in the limit of small rapidity separation. While transplanckian gravitational scattering can be perturbatively calculated, black-hole formation occurs in the regime in which gravitational interactions are strong. 


\begin{tabular}{|c|c|c|c|}
\hline Experiment & Process & + & - \\
\hline LEP [7] & $e^{+} e^{-} \rightarrow \gamma \gamma$ & $0.93 \mathrm{TeV}$ & $1.01 \mathrm{TeV}$ \\
\hline LEP $[8-11]$ & $e^{+} e^{-} \rightarrow e^{+} e^{-}$ & $1.18 \mathrm{TeV}$ & $1.17 \mathrm{TeV}$ \\
\hline H1 [12] & $e^{+} p$ and $e^{-} p$ & $0.74 \mathrm{TeV}$ & $0.71 \mathrm{TeV}$ \\
\hline ZEUS [13] & $e^{+} p$ and $e^{-} p$ & $0.72 \mathrm{TeV}$ & $0.73 \mathrm{TeV}$ \\
\hline $\mathrm{CDF}[14]$ & $p \bar{p} \rightarrow e^{+} e^{-}, \gamma \gamma$ & $0.99 \mathrm{TeV}$ & $0.96 \mathrm{TeV}$ \\
\hline $\mathrm{D} \varnothing[14]$ & $p \bar{p} \rightarrow e^{+} e^{-}, \gamma \gamma$ & $1.28 \mathrm{TeV}$ & $1.14 \mathrm{TeV}$ \\
\hline $\mathrm{D} \varnothing[15]$ & $p \bar{p} \rightarrow j j$ & $1.48 \mathrm{TeV}$ & $1.48 \mathrm{TeV}$ \\
\hline CMS at $7 \mathrm{TeV}$ with $40 / \mathrm{pb}$ [16] & $p p \rightarrow \mu^{-} \mu^{+}$ & $1.6 \mathrm{TeV}$ & $1.6 \mathrm{TeV}$ \\
\hline CMS at $7 \mathrm{TeV}$ with $36 / \mathrm{pb}$ [30] & $p p \rightarrow \gamma \gamma$ & $1.74 \mathrm{TeV}$ & $1.71 \mathrm{TeV}$ \\
\hline ATLAS at $7 \mathrm{TeV}$ with $3.1 / \mathrm{pb}$ & $p p \rightarrow j j$ & $2.2 \mathrm{TeV}$ & $2.1 \mathrm{TeV}$ \\
\hline ATLAS at $7 \mathrm{TeV}$ with $36 / \mathrm{pb}$ & $p p \rightarrow j j$ & $4.2 \mathrm{TeV}$ & $3.2 \mathrm{TeV}$ \\
\hline CMS at $7 \mathrm{TeV}$ with $36 / \mathrm{pb}$ & $p p \rightarrow j j$ & $4.2 \mathrm{TeV}$ & $3.4 \mathrm{TeV}$ \\
\hline
\end{tabular}

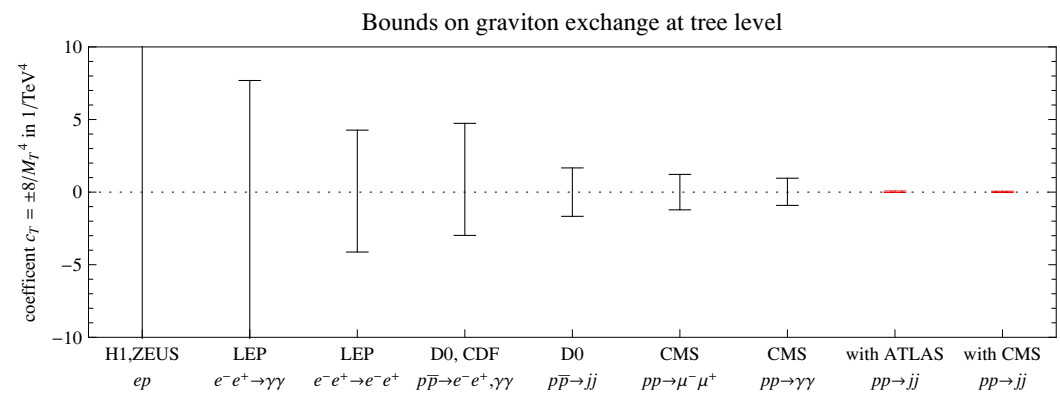

Table 1. Tree-level graviton exchange: 95\% CL limits on the coefficient $M_{T}$ (known as Hewett normalization [4]) of the dimension-8 operator $\mathcal{T}$ of eq. (1.2) for positive and negative interference. The last three limits are derived in this work.

Furthermore brane fluctuations (massless 'branons') give rise to the same effect 1 (as in $\delta=6$ ) and 2 (as in $\delta=4$ ) [25]. In its first stage with low statistics, LHC is particularly sensitive to the operator in eq. (1.2), because its high dimensionality means that the high energy of the LHC collisions is the key factor.

In section 2 we show that the present low-statistics data about $p p \rightarrow j j$ already set a bound on the coefficient $8 / M_{\mathcal{T}}^{4}$ of the effective operator (1.2) which is significantly stronger than those obtained from any previous experiment, as summarized in table 1 . In section 3 we discuss how $M_{\mathcal{T}}$ can be related to $M_{D}$ and $\delta$, and derive explicit expressions for the full graviton-exchange amplitude, including both gravitons at the ultraviolet end of the spectrum and gravitons that can be produced at LHC. In section 4 we compare the full amplitude to LHC data. Section 5 contains our conclusions.

\section{Fit to the graviton-exchange effective operator}

We compare the first LHC data to the new physics described by eq.s (1.2) and (1.3). Since the $\delta$-dependent double trace term in $\mathcal{T}$ is irrelevant for collisions of particles with masses 


\begin{tabular}{|c|c|cc|}
\hline Experiment & Process & + & - \\
\hline LEP combined [19] & $e^{+} e^{-} \rightarrow e^{+} e^{-}$ & 11.3 & 11.5 \\
LEP combined [19] & $e^{+} e^{-} \rightarrow \mu^{+} \mu^{-}$ & 16.4 & 12.7 \\
LEP combined [19] & $e^{+} e^{-} \rightarrow \ell^{+} \ell^{-}$ & 17.2 & 15.1 \\
LEP combined [19] & $e^{+} e^{-} \rightarrow b \bar{b}$ & 15.3 & 11.5 \\
H1 [12] & $e^{+} p$ and $e^{-} p$ & 2.5 & 3.9 \\
ZEUS [13] & $e^{+} p$ and $e^{-} p$ & 4.6 & 5.3 \\
DØ [20] & $p \bar{p} \rightarrow e^{+} e^{-}$ & 4.7 & 5.5 \\
CDF [20] & $p \bar{p} \rightarrow \ell^{+} \ell^{-}$ & 4.5 & 5.6 \\
CCFR [21] & $\nu N$ scattering & 3.7 & 5.9 \\
DØ [20] & $p \bar{p} \rightarrow j j$ & 3.2 & 3.1 \\
ATLAS at 7 TeV with 3.1/pb & $p p \rightarrow j j$ & 5.3 & 4.2 \\
CMS at 7 TeV with 36/pb & $p p \rightarrow j j$ & 11 & 8.1 \\
\hline \multicolumn{2}{|c|}{ combined } & 22.4 & 15.7 \\
\hline
\end{tabular}

Table 2. Loop-level graviton exchange: 95\% CL limits on the coefficient $\left|c_{\Upsilon} / 4 \pi\right|^{-1 / 2}$ (in TeV) of the dimension- 6 operator $\Upsilon$ of eq. (1.3) for positive and negative values of $c_{\Upsilon}$.

much smaller than the LHC energy, our subsequent analysis applies to any number of extra dimensions (larger than 2) as well as to branon effects.

The tree-level exchange of virtual gravitons described by the Lagrangian of eq. (1.2) mediates the processes

$$
p p \rightarrow \ell^{+} \ell^{-}, \quad p p \rightarrow \gamma \gamma, \quad p p \rightarrow j j .
$$

The experimental collaborations concentrated their sensitivity studies on the di-lepton and di-photon final states. However the corresponding cross sections are significantly lower than the $p p \rightarrow j j$ cross section, and this is the main factor that determines the observability of these signals at the initial LHC stage with $\sqrt{s}=7 \mathrm{TeV}$ and low luminosity. Indeed requiring final states with invariant mass greater than $1 \mathrm{TeV}$, jets, leptons and photons with $\eta<2.5$, and additionally requiring $\left|\eta_{1}-\eta_{2}\right|<1.2$ for the jets, we find

$$
\sigma=\left(\frac{2 \mathrm{TeV}}{M_{T}}\right)^{8} \times\left\{\begin{array}{l}
12.5 \mathrm{pb} \text { for } p p \rightarrow j j \\
10.4 \mathrm{fb} \text { for } p p \rightarrow \mu^{+} \mu^{-} \\
21.3 \mathrm{fb} \text { for } p p \rightarrow \gamma \gamma
\end{array} .\right.
$$

This large difference in cross sections is due partly to trivial flavor and color factors, and partly to the fact that the processes are mediated by the operator of dimension 8 in eq. (1.2), which gives larger rates for the channels with more energetic initial states. In particular $p p \rightarrow j j$ benefits from the high energy of the initial partons $u u$ in the $t$-channel process.

In the following we shall show that, from the angular distribution of the jets, even with only $3.1 \mathrm{pb}^{-1}$ of integrated luminosity it was possible to obtain the dominant limit to date on the operator $\mathcal{T}$. 

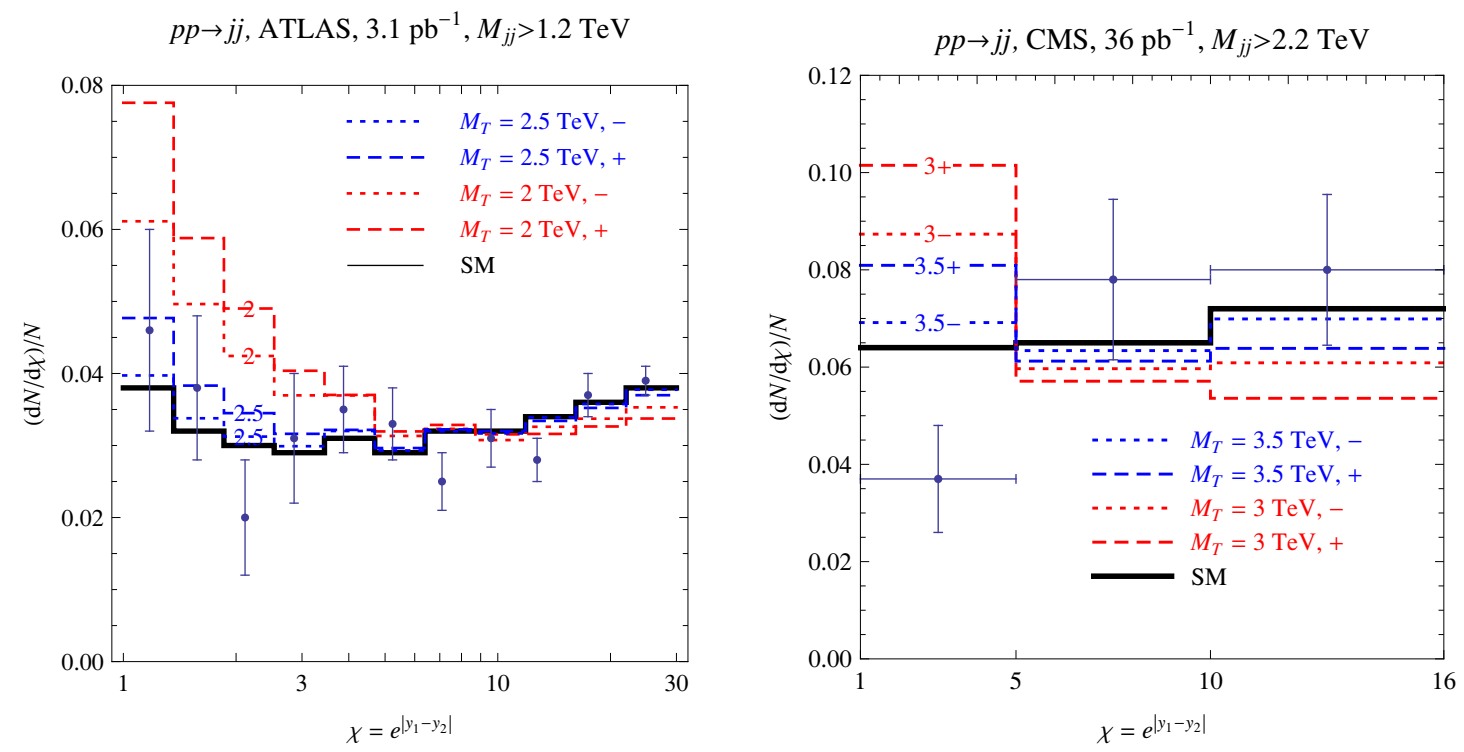

Figure 2. Left (right): $p p \rightarrow j j$ angular distribution at ATLAS with $M_{j j}>1.2 \mathrm{TeV}$ (at CMS with $M_{j j}>2.2 \mathrm{TeV}$ ) binned as a function of the angular distance $\chi$. The experimental data (crosses) are compared to the SM prediction (black histogram) and to the expectation including virtual graviton effects at tree level.

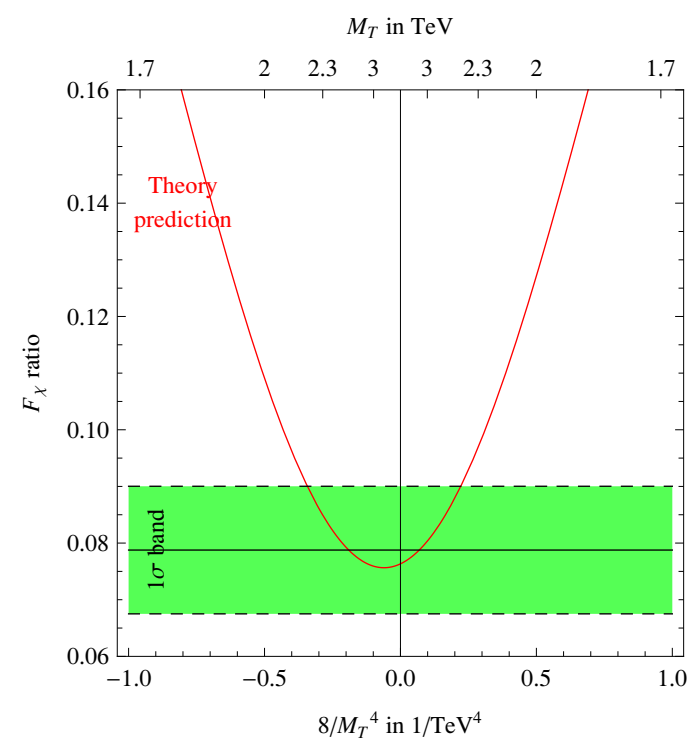

Figure 3. Experimental values from ATLAS and theoretical values of the variable $F_{\chi}$ (fraction of $j j$ events with $M_{j j}>1.2 \mathrm{TeV}$ in the central region).

We study the effect of graviton-mediated amplitudes in the differential and in the total cross section, including interference effects between the SM and the new contributions. Both the total and the differential cross section are affected by NLO effects. However this sensitivity to higher order effects can be reduced by choosing a suitable kinematical quantity and restricting the analysis to certain kinematical regions. 
ATLAS [26] and CMS [17, 18, 27] have searched for the effect of contact interactions in the angular distribution of dijet events. Both collaborations have studied the centrality ratio distribution, and ATLAS also released the normalized distribution in several ranges of invariant mass of the jets on the variable

$$
\chi \equiv \exp \left|y_{1}-y_{2}\right|,
$$

where $y_{1,2}$ are the two jet rapidities. Due to the dominance of Coulomb-like scattering in the SM, these distributions are expected to be almost flat in the case of QCD, which helps to reduce the impact of smearing effects. Contact interactions, especially those in eq. (1.2) being mediated by a spin-2 particle, have a different angular distribution with respect to QCD and result in a deviation from a flat distribution.

Data for the $\chi$ distribution from ATLAS are reported in figure 2a together with the SM expectation at next-to-leading order [26]. Figure2a shows also the effect of the graviton operator $\mathcal{T}$ for $M_{T}=2$ and $2.5 \mathrm{TeV}$ for both positive or negative interference with the SM.

The prediction of the effect of the operator $\mathcal{T}$ has been obtained simulating the effect of this operator at the partonic level with MADGRAPH [28, 29] and CTEQ6L parton distribution functions. We checked that showering and detector effects do not alter significantly the prediction. In particular we checked that with the current uncertainties on the data the limit on the contact interaction studied by ATLAS [26] is reproduced at the partonic level within $20 \%$.

We compare data with the theoretical expectation and we compute the 95\% CL bound on the coefficient of the $\mathcal{T}$ operator by imposing

$$
\chi^{2}=\sum_{i}^{\text {bins }} \frac{\left(t_{i}\left(c_{\mathcal{T}}\right)-\mu_{i}\right)^{2}}{\sigma_{i \text { stat }}^{2}+\sigma_{\text {syst }}^{2}}<\chi_{\text {min }}^{2}+3.84,
$$

where $\mu_{i}$ are the experimental central values, $\sigma_{i \text { stat }}$ the statistical errors, $\sigma_{\text {syst }} \approx 0.003$ estimates the systematic uncertainties (we ignore possible correlations between different bins) which are presently subdominant and $t_{i}\left(c_{\mathcal{T}}\right)$ are the theoretical predictions, computed for some values of $c_{\mathcal{T}}$ and fitted in each bin as a quadratic function of $c_{\mathcal{T}}=8 / M_{T}^{4}$. We find the bound $M_{T}>2.1 \mathrm{TeV}$ reported in table 1 . This significantly exceeds all previous bounds.

ATLAS [26] reports also the observation on the quantity $F_{\chi}$, defined as the ratio between the events in the first four $\chi$ bins $(\chi<3.3)$ with respect to the total $621 j j$ events in the acceptance region. The present experimental value is $F_{\chi}=0.078 \pm 0.011$, to be compared to the SM prediction at NLO, $F_{\chi}^{\mathrm{SM}}=0.076$ [26]. We find that the variable $F_{\chi}$ captures well the effect of contact interactions, as it corresponds to comparing the crosssection in the central region for the SM and the contact interaction. Indeed the bound on $M_{T}$ negligibly changes going from the full fit to the one-variable $F_{\chi}$ fit, as illustrated in figure 3 .

The variable $F_{\chi}$ allows us to easily estimate how the sensitivity to $M_{T}$ improves with higher luminosity. Assuming that the measurement is dominated by the statistical error we find that with the luminosity of about $50 \mathrm{pb}^{-1}$ currently collected by the LHC experiments the expected $95 \%$ CL limit on $M_{T}$ is about $3 \mathrm{TeV}$. 

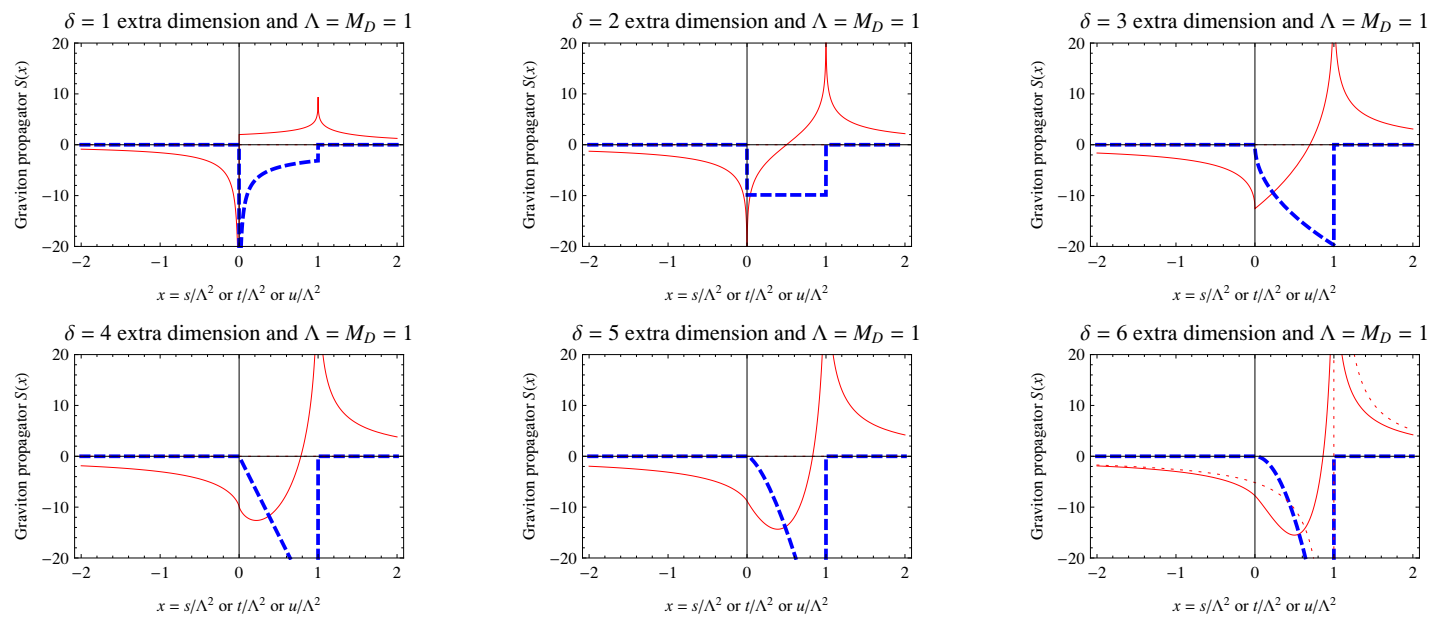

Figure 4. Real part (solid red curve) and imaginary part (dashed blue curve) of $\mathcal{S}(x)$ in units $\Lambda=M_{D}=1$. The dotted line in the $\delta=6$ panel shows the single-pole approximation (one graviton with mass $\Lambda$ ) that holds in the limit $\delta \rightarrow \infty$.

CMS $p p \rightarrow j j$ data after $36 \mathrm{pb}^{-1}$ have been recently presented $[17,18]$ and are here plotted in figure $2 \mathrm{~b}$. We can reliably estimate the resulting bound, $M_{T}>3.4 \mathrm{TeV}$, as reported in table 1. This is comparable to the sensitivity, $3.2 \mathrm{TeV}$, despite the apparent mild statistical fluctuation in the first bin. From ATLAS $p p \rightarrow j j$ data after $36 \mathrm{pb}^{-1}[32]$ we estimate $M_{T}>3.2 \mathrm{TeV}$.

We can compare the sensitivity of the dijet channel to those of the $p p \rightarrow \ell^{+} \ell^{-}$and $p p \rightarrow \gamma \gamma$ channels considered by the experimental collaborations. CMS [30] finds $M_{T}>$ $1.8 \mathrm{TeV}$ from $p p \rightarrow \gamma \gamma$ after $36 / \mathrm{pb}$ of integrated luminosity. Ref. [30] reports a 95\% C.L. sensitivity in the $\gamma \gamma$ channel to $M_{T} \simeq 3 \mathrm{TeV}$ for more than $150 \mathrm{pb}^{-1}$ at $10 \mathrm{TeV}$ center of mass energy and [31] claims a sensitivity of the leptonic channel to $M_{T} \simeq 3 \mathrm{TeV}$ with 100 $\mathrm{pb}^{-1}$ of $14 \mathrm{TeV}$ data. The proposed measurements essentially consist in counting events with large invariant mass, as in eq. (2.2). The $p p \rightarrow j j$ signal already reached the same sensitivity with current center of mass energy of $7 \mathrm{TeV}$ and current $36 \mathrm{pb}^{-1}$ of integrated luminosity, and will remain the most sensitive channel until systematic uncertainties will dominate the error on the measured angular distribution.

Finally, we computed the bound on the dimension- 6 operator $\Upsilon$ of eq. (1.3) generated by graviton exchange at loop level. The result is shown in table 2 together the other existing bounds. With the published data we find a bound from dijets at LHC that is comparable to the bound from Tevatron and strongly subdominant with respect to the bound from LEP. Even with the data with $36 \mathrm{pb}^{-1}$ of integrated luminosity we get a bound subdominant with respect to LEP, although significantly larger that existing limits from Tevatron.

\section{Tree-level graviton exchange}

In view of its experimental significance, we reconsider the theory behind eq. (1.2) and the approximation of tree-level graviton exchange with an effective operator. In full generality, 
tree-level graviton-exchange (figure 1a) leads to a scattering amplitude of the form

$$
\mathcal{A}=\mathcal{S}(s)\left(T_{\mu \nu} T^{\mu \nu}-\frac{T_{\mu}^{\mu} T_{\nu}^{\nu}}{\delta+2}\right)
$$

The function $\mathcal{S}$ is obtained by summing over all the Kaluza-Klein (KK) tower of gravitons. As will be discussed below, if the typical energy resolution of the experiment is broader than the mass separation between two KK states, the sum can be approximated as an integral over the extra-dimensional momentum $q$ of the graviton. Such integral is UV divergent for $\delta>1$ extra dimensions. So we regularize the integral by including only KK excitations with mass $m=|q|$ below an arbitrary cut-off $\Lambda$, which parametrizes the onset of the unknown quantum-gravity physics. A small (large) ratio $\Lambda / M_{D}$ effectively means that quantum gravity is weakly (strongly) coupled [5]. The use of the cutoff allows for a comparison of the experimental limits on the operator (1.2) with the searches for real graviton emission in missing $p_{T}$ events. Cutting off the integral, we find

$$
\mathcal{S}(s)=\frac{1}{M_{D}^{2+\delta}} \int_{|q|<\Lambda} \frac{d^{\delta} q}{s-q^{2}+i \varepsilon}=\frac{\pi^{\delta / 2} \Lambda^{\delta-2}}{\Gamma(\delta / 2) M_{D}^{2+\delta}} F_{\delta}\left(\frac{s}{\Lambda^{2}}\right)
$$

where $\Gamma$ is the Euler function and $F_{\delta}$ is recursively defined as

$$
F_{\delta+2}(x)=x F_{\delta}(x)-\frac{2}{\delta}
$$

and $^{1}$

$$
F_{1}(x)=\frac{2}{\sqrt{x}} \operatorname{arctanh} \frac{1}{\sqrt{x}}, \quad F_{2}(x)=-\log \left(1-\frac{1}{x}\right) .
$$

Figure 4 shows the behavior of the real (solid line) and imaginary (dashed line) parts of $\mathcal{S}$, for various values of $\delta$. In the case of $t$-channel exchange, the variable of the function $\mathcal{S}$ is negative and no imaginary part is developed, since the exchanged graviton cannot be on-shell. For $\delta>2$ the integral is dominated by the heaviest graviton with mass $m \approx \Lambda$ and thus, for $s \ll \Lambda^{2}$, the function $\mathcal{S}$ can be treated as a constant with no momentum dependence and the scattering amplitude can be approximated by the effective operator $\mathcal{T}$ of eq. (1.2) with a coefficient which is usually defined as [4]

$$
\mathcal{S}\left(s \ll \Lambda^{2}\right)= \begin{cases}\frac{\pi^{\delta / 2}}{(1-\delta / 2) \Gamma(\delta / 2)} \frac{\Lambda^{\delta-2}}{M_{D}^{\delta+2}} \equiv \frac{8}{M_{\mathcal{T}}^{4}} & \text { for } \delta>2 \\ \frac{\pi}{M_{D}^{4} \ln \frac{s}{\Lambda^{2}}} & \text { for } \delta=2 \\ \frac{-i \pi}{M_{D}^{3} \sqrt{s}} & \text { for } \delta=1\end{cases}
$$

${ }^{1}$ Equivalent, but more explicit, expressions for $F_{1,2}$ are

$$
\begin{aligned}
& \operatorname{Im} F_{1}=-\pi / \sqrt{x}, \quad \operatorname{Im} F_{2}=-\pi \quad \text { for } 0<x<1 \text { and zero otherwise } \\
& \operatorname{Re} F_{1}=\left\{\begin{array}{ll}
\frac{1}{\sqrt{x}} \ln \left|\frac{\sqrt{x}+1}{\sqrt{x}-1}\right| & \text { for } x>0 \\
\frac{1}{\sqrt{-x}}[2 \arctan (\sqrt{-x})-\pi] & \text { for } x<0
\end{array} \quad \operatorname{Re} F_{2}=-\ln \left|1-\frac{1}{x}\right| .\right.
\end{aligned}
$$


However, in view of the high dimensionality of the operator, the dominant LHC bound comes from the highest energy events, and it is appropriate to retain the full amplitude, including the dependence on the cut-off $\Lambda$.

We would like now to comment on the validity of approximating the sum over virtual gravitons with an integral.

\section{$3.1 \delta=1$}

It is well known that gravity at macroscopic scales and astrophysical considerations strongly constrain the cases $\delta=1,2$, and 3 . The corresponding fundamental mass $M_{D}$ can lie around the weak scale only if the theory is modified in the infrared. This can be achieved by introducing a warping factor [33] with a small mass parameter $\mu$ (of a few $\mathrm{MeV}$ ) which lifts the lightest KK mode of the graviton (and characterizes the KK graviton mass splitting, since $m_{n} \simeq \pi n \mu$ for $n \gg 1$ ), without modifying the UV behavior of the theory and its collider predictions [34].

Let us first consider the case $\delta=1$, in which the KK summation can be explicitly performed with the result $[35,36]$

$$
\begin{aligned}
\mathcal{S}(s) & =\frac{1}{\Lambda_{\pi}^{2}} \sum_{n} \frac{1}{s-m_{n}^{2}+i m_{n} \Gamma_{G}\left(m_{n}\right)}=-\frac{\pi}{M_{5}^{3} \sqrt{s}} K \\
K & =\frac{\sin 2 A+i \sinh 2 \epsilon}{2\left(\cos ^{2} A+\sinh ^{2} \epsilon\right)} \quad A=\pi\left(\frac{\sqrt{s}}{\Delta m}+\frac{1}{4}\right) \quad \epsilon=\left.\frac{\pi \Gamma_{G}}{2 \Delta m}\right|_{m=\sqrt{s}} .
\end{aligned}
$$

Here $\Lambda_{\pi}$ is the interaction scale of individual gravitons, related to the fundamental mass of the 5 -dimensional theory $M_{5}$ by [34]

$$
\Lambda_{\pi}^{2}=\frac{M_{5}^{3}}{2 \pi \mu}
$$

The mass splitting between KK gravitons $\Delta m$ and the decay width of the $n$-th KK graviton $\Gamma_{G}\left(m_{n}\right)$ are given by

$$
\Delta m=\pi \mu \quad \Gamma_{G}\left(m_{n}\right)=\frac{c m_{n}^{3}}{\pi \Lambda_{\pi}^{2}}
$$

where $c=1 / 80,1 / 320$, and $1 / 960$ for graviton decays into a massless vector, Weyl fermion, and conformally-coupled real scalar, respectively [3]. Consequently, we find $c=283 / 960$ after summing over all SM particles. The parameter $\epsilon$ in eq. (3.8), which measures the relative separation of the individual graviton resonances $(\epsilon \ll 1$ means well separated resonances, $\epsilon \gtrsim 1$ means overlapping resonances), is given by

$$
\epsilon=c\left(\frac{\sqrt{s}}{M_{5}}\right)^{3}
$$

Therefore $\epsilon$ remains finite in the limit $\mu \rightarrow 0$, which corresponds to sending the compactification volume to infinity $\left(M_{\mathrm{Pl}} \rightarrow \infty\right)$.

The expression of $\mathcal{S}$ in eq. (3.7) is a rapidly oscillating function. However, we are interested in the case in which the energy spread of the initial and final states is broader 
than the mass separation $\mu$. It is then convenient to average eq. (3.7) within one oscillation period, obtaining the smoothly varying function ${ }^{2}[34]$

$$
\langle\mathcal{S}\rangle=-\frac{i \pi}{M_{5}^{3} \sqrt{s}} .
$$

We can now take an alternative approach and work directly in the continuum, by replacing the discrete KK summation with an integral ${ }^{3}$

$$
\mathcal{S}(s)=\frac{1}{\Lambda_{\pi}^{2}} \int \frac{d m}{\pi \mu} \frac{1}{s-m^{2}+i m \Gamma_{G}(m)} \stackrel{\Gamma_{G \rightarrow 0}}{\simeq}-\frac{i \pi}{M_{5}^{3} \sqrt{s}} .
$$

Therefore, the procedure of integrating in the continuum, eq. (3.13), gives exactly the same result as the averaged summation in eq. (3.12). This shows that, as long as the energy resolution is broader than the mass separation, it is perfectly adequate to treat virtual gravitons as a continuum.

Let us now consider the modulus square of the expression in eq. (3.7), averaged over an oscillation period ${ }^{4}$

$$
\left\langle|\mathcal{S}|^{2}\right\rangle=\frac{\pi^{2}}{M_{5}^{6} s}\left(1+\frac{4}{e^{4 \epsilon}-1}\right) \stackrel{\epsilon \rightarrow 0}{\simeq} \frac{1}{\epsilon}\left(\frac{\pi}{M_{5}^{3} \sqrt{s}}\right)^{2} .
$$

While for $\epsilon>1$ we find $\left\langle|\mathcal{S}|^{2}\right\rangle \simeq|\langle\mathcal{S}\rangle|^{2}$, in the relevant case of small $\epsilon$ we obtain that eq. (3.14) leads to an enhancement of a factor $1 / \epsilon$. Note that the enhanced term in eq. (3.14) has a lower order in powers of graviton coupling constants than expected for a scattering process, because it corresponds to the production of real gravitons.

The same result can be obtained also by calculating $|\mathcal{S}|^{2}$ in the continuum. If we are interested in the real production of well-separated narrow resonances, we can neglect interference effects. Then the calculation in the continuum, for $\epsilon<1$, gives ${ }^{5}$

$$
|\mathcal{S}|^{2}=\frac{1}{\Lambda_{\pi}^{4}} \int \frac{d m}{\pi \mu} \frac{1}{\left(s-m^{2}\right)^{2}+m^{2} \Gamma_{G}^{2}} \stackrel{\Gamma_{G} \rightarrow 0}{\simeq} \frac{1}{\epsilon}\left(\frac{\pi}{M_{5}^{3} \sqrt{s}}\right)^{2} .
$$

The result of the calculation in the continuum agrees with the discrete summation in eq. (3.14), when initial and final particle states are spread in energy more than the KK mass separation.

\footnotetext{
${ }^{2}$ We use$$
\frac{1}{2 \pi} \int_{0}^{2 \pi} d x \frac{\sin 2 x+a}{\cos ^{2} x+b}=\frac{a}{\sqrt{b(1+b)}} .
$$

${ }^{3}$ We use

$$
\lim _{\epsilon \rightarrow 0} \frac{1}{x+i \epsilon}=P\left(\frac{1}{x}\right)-i \pi \delta(x)
$$

${ }^{4}$ We use

$$
\frac{1}{2 \pi} \int_{0}^{2 \pi} d x \frac{\sin ^{2} 2 x+a}{\left(\cos ^{2} x+b\right)^{2}}=\left[2+\frac{a}{4 b(1+b)}\right] \frac{1+2 b}{\sqrt{b(1+b)}}-4
$$

${ }^{5}$ We use

$$
\lim _{\epsilon \rightarrow 0} \frac{\epsilon}{x^{2}+\epsilon^{2}}=\pi \delta(x)
$$
}


As mentioned above, the Feynman diagram in figure 1a includes two effects: a) $2 \rightarrow 2$ scattering processes mediated by virtual gravitons, and b) $2 \rightarrow 1 \rightarrow 2$ production of one graviton KK resonance with mass equal to $\sqrt{s}$ that eventually decays into SM particles. The enhancement in eq. (3.14) is the contribution from process b). In the $\delta=1$ scenario we are considering, the graviton decays well inside the detector, such that process b) must be included and there are no missing-energy signals (a point missed in previous works on the topic).

On the contrary, in the $\delta>1$ scenarios considered in the next section, KK gravitons typically decay far away from the detectors, such that process b) does not contribute to $2 \rightarrow 2$ scatterings observed at LHC.

\section{$3.2 \delta>1$}

The previous result can be generalized to $\delta>1$. The amplitude smoothed over scattering wave packets broader than the mass splitting between KK gravitons is obtained by replacing the discrete summation with an integral

$$
\mathcal{S}(s)=\frac{1}{M_{\mathrm{Pl}}^{2}} \sum_{i} \frac{1}{s-m_{i}^{2}+i m_{i} \Gamma_{G}\left(m_{i}\right)} \rightarrow \frac{2 \pi^{\delta / 2}}{\Gamma(\delta / 2) M_{D}^{2+\delta}} \int_{0}^{\Lambda} d m \frac{m^{\delta-1}}{s-m^{2}+i m \Gamma_{G}(m)},
$$

where $M_{\mathrm{Pl}}$ is the reduced Planck mass. Writing the graviton propagator in the narrowwidth approximation and using the relation in footnote 3 , we obtain an expression for $\langle\mathcal{S}\rangle$ that is identical to eq. (3.2).

For generic $\delta$, the graviton width is $\Gamma_{G}(m)=c m^{3} / \pi M_{\mathrm{Pl}}^{2}$ and the mass difference is

$$
\Delta m=\frac{\Gamma(\delta / 2) M_{D}^{2+\delta}}{2 \pi^{\delta / 2} M_{\mathrm{Pl}}^{2} m^{\delta-1}} .
$$

Here we are considering the case in which the KK graviton spectrum is not distorted in the infrared $(\mu=0)$. Analogously to the $\delta=1$ case, we can define

$$
\left.\epsilon \equiv \frac{\pi \Gamma_{G}}{2 \Delta m}\right|_{m=\sqrt{s}}=\frac{\pi^{\delta / 2} c}{\Gamma(\delta / 2)}\left(\frac{\sqrt{s}}{M_{D}}\right)^{2+\delta} .
$$

Note that $\epsilon<1$ as long as the low-energy effective theory can be trusted $\left(\sqrt{s}<M_{D}\right)$, showing that the graviton resonances are narrow and well separated. Using the narrowwidth approximation (see relation in footnote 4) we find that the leading contribution is

$$
\left\langle|\mathcal{S}(s)|^{2}\right\rangle=\frac{(\operatorname{Im} \mathcal{S})^{2}}{\epsilon} .
$$

As before, this term has to be interpreted as the production of a graviton with mass $\sqrt{s}$. Since the graviton decays well beyond the detector, this term contributes to "missing energy" and not to the signal we are considering and should be subtracted from the final result. Effectively, the rate of interest is obtained by taking the modulus square of eq. (3.2). The situation can be different in intermediate scenarios with $\mu>0$ and shorter graviton life-time; a life-time comparable to the detector size would lead to $2 \rightarrow 2$ signals with displaced-vertex. 

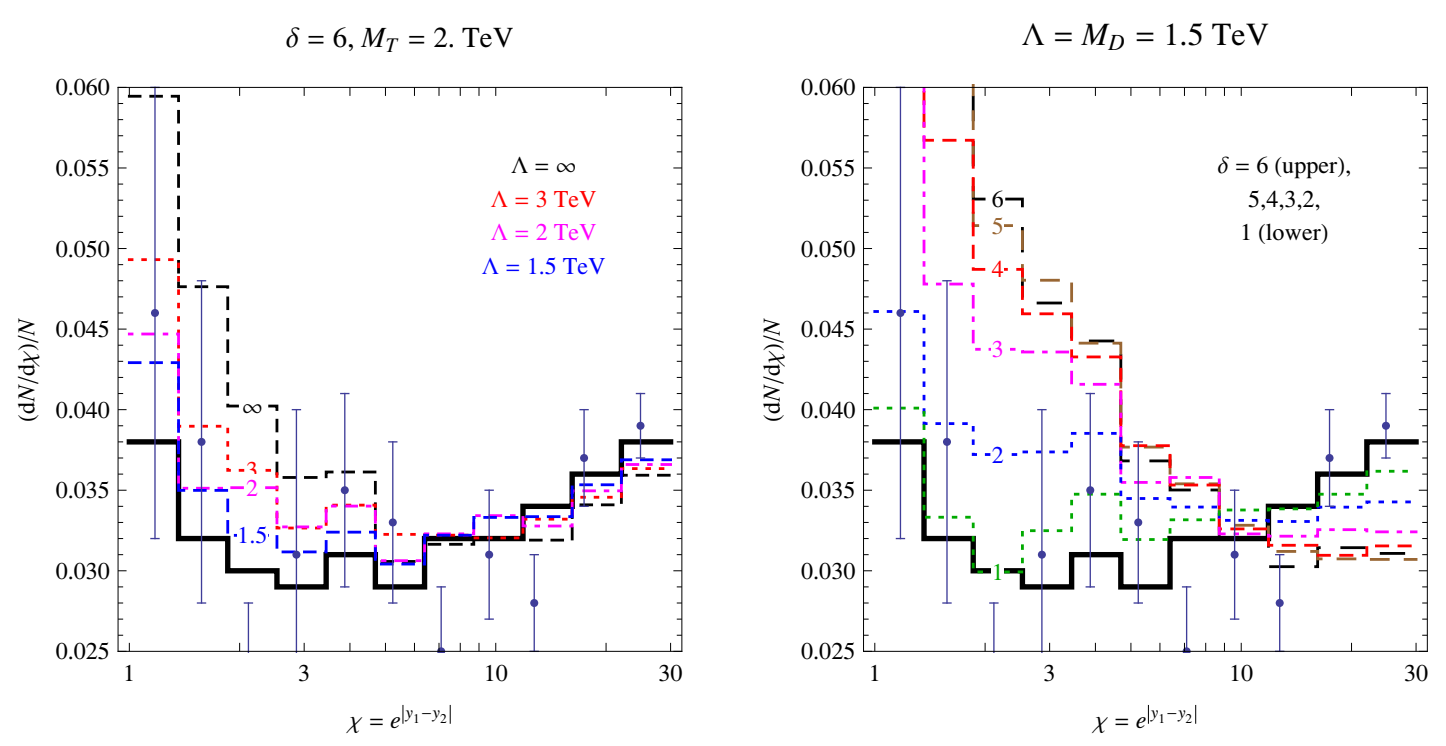

Figure 5. Left: $p p \rightarrow j j$ angular distribution for fixed $\delta=6, M_{T}=2 \mathrm{TeV}, M_{j j}>1.2 \mathrm{TeV}$ and different values of $\Lambda$ (as indicated) and consequently of $M_{D}$. The effective-operator $\mathcal{T}$ is formally reproduced in the limit $\Lambda \rightarrow \infty$. Right: dependence on the number $\delta$ of extra dimensions at fixed $M_{D}=\Lambda=1.5 \mathrm{TeV}$. The data are from ATLAS [26].

\section{Fit to the full graviton-exchange amplitude}

Formulæ for the cross sections from tree-level graviton effects in any number of extra dimensions can be found in the appendix of ref. [34]. We implement them in Pythia8 [37] and verify that in the effective-operator approximation $\left(\mathcal{S}=8 / M_{T}^{4}\right)$ the various distributions reproduce the ones previously obtained with MADGRAPH and that hadronization and jet reconstruction negligibly affect the observables we consider.

We can now compare the data with the full graviton-exchange amplitude, computed in terms of the cut-off $\Lambda$, defined to be the maximal KK graviton mass. Even for $\delta=1$ the correct treatment of $|\mathcal{S}|^{2}$ in the $s$-channel is numerically irrelevant for this work, where we consider the $p p \rightarrow j j$ signal which is dominated by the $u u$ initial state which has no $s$-channel.

Figure 5a shows how the theoretical prediction changes with the cut-off $\Lambda$ keeping fixed the coefficient of the effective operator $\mathcal{T}$ to be $M_{T}=2 \mathrm{TeV}$, around the present bound: the full amplitude must be used unless $\Lambda \gg M_{D}$. Figure5b shows how the theoretical prediction changes with $\delta$ keeping fixed $M_{D}$ and $\Lambda$.

The results of our fit are shown in figure 6 , as functions of $M_{D}$ and of the ratio $\Lambda / M_{D}$. The $95 \%$ CL bound is defined as $\chi^{2}<\chi_{\mathrm{SM}}^{2}+3.84$. As previously discussed, the ratio $\Lambda / M_{D}$ effectively parameterizes the unknown strength of the full quantum-gravity theory. The gray area at larger $\Lambda / M_{D}$ covers the region estimated to be non-perturbative according to naive dimensional analysis [5].

The shaded area covers the region excluded by the angular distribution at $M_{j j}>$ 1.2 TeV. The dashed curve also shows the exclusion obtained considering only the $F_{\chi}$ 

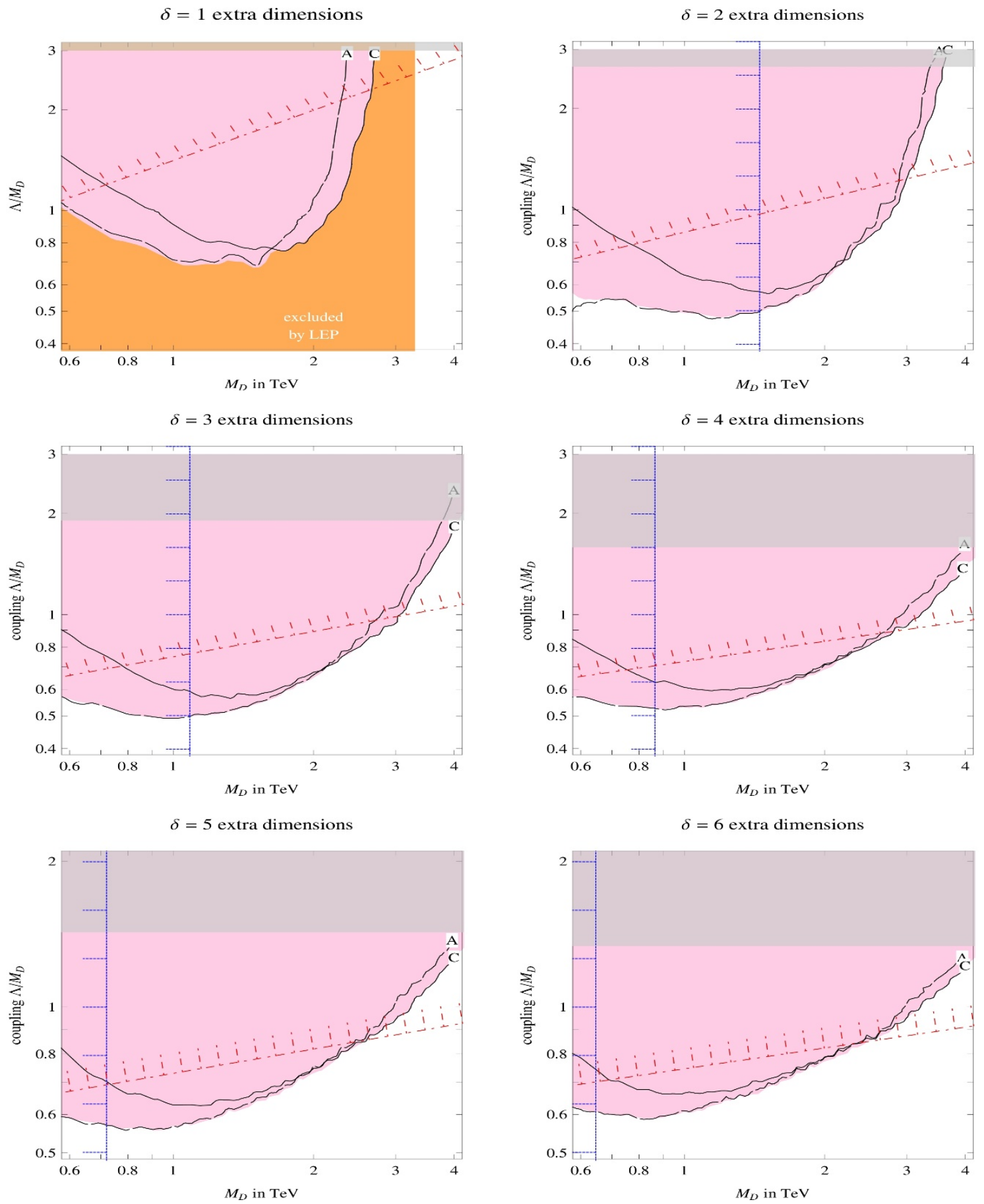

Figure 6. The shaded area is the bound from virtual graviton exchange at CMS (continuous line denoted as ' $\mathrm{C}$ ', data after $36 / \mathrm{pb}$ ), ATLAS (long-dashed line denoted as 'A', data after $36 / \mathrm{pb}$ ). Vertical blue line: bound from graviton emission (as summarized in table 1 of [5]). Red line: Naive Dimensional Analysis estimate of LEP bound from loop graviton exchange. Upper shading: NDA estimate of the non-perturbative region. 
ratio: it gives a good approximation to the full fit in the region with larger $\Lambda$ where the effective operator approximation is valid; but fails in the region with lower $\Lambda$.

For comparison, the other two lines show:

- the combined Tevatron-LEP bound from graviton emission (vertical blue lines; computed ignoring the dependence on $\Lambda$ ).

- the LEP bound on loop graviton exchange (red line), estimated according to naive dimensional analysis.

For $\delta=1$ the LHC bound $M_{D} \gtrsim 1.5 \mathrm{TeV}$ remains subdominant with respect to the bound from $e^{-} e^{+} \rightarrow f \bar{f}$ scatterings at LEP2, that we estimate to be $M_{D} \gtrsim 3.4 \mathrm{TeV}$. For $\delta>1$ already the first ATLAS data at $3.1 \mathrm{pb}^{-} 1$ explore new regions of the parameters space of gravity in extra dimensions. The new data at $36 \mathrm{pb}^{-} 1$ provide stronger bounds.

\section{Conclusion}

We found that the very first LHC data about $p p \rightarrow j j$, despite the low statistics and the uncertainties intrinsic in the hadronic nature of the final state, improve significantly previous bounds on the coefficient of the effective dimension- 8 operator $\mathcal{T}$ generated by virtual graviton or branon exchange, and predicted by theories with extra dimensions. This arises thanks to the high dimensionality of the operator, which rewards the higher energy of LHC with respect to previous colliders.

In a second part of the work we went beyond the effective-operator approximation and computed the full amplitude generated by tree level graviton exchange in terms of a cut-off parameter $\Lambda$, which is the maximal KK graviton mass. We clarified that the enhanced effect of lighter gravitons that can be produced on-shell must be included only when such gravitons decay within the detector. Figure6 shows the resulting LHC bounds in the $\left(M_{D}, \Lambda / M_{D}\right)$ plane.

\section{Acknowledgments}

We thank Riccardo Rattazzi for discussions about the physics of extra-dimensions and for his suggestions. We are grateful to Georgios Choudalakis and Frederik Ruehr for discussions about the published results of ATLAS, and to Greg Landsberg about CMS data presented in $[17,18]$. RF is also grateful to Diego Blas and Michele Redi for many discussions on gravity and extra-dimensions. This work was supported by the ESF grant MTT8 and by SF0690030s09 project. The work of RF is supported by the Swiss National Science Foundation under contract No. 200021-116372. The work of PL is supported in part by the European Programme 'Unification in the LHC Era', contract PITN-GA-2009237920 (UNILHC).

Open Access. This article is distributed under the terms of the Creative Commons Attribution Noncommercial License which permits any noncommercial use, distribution, and reproduction in any medium, provided the original author(s) and source are credited. 


\section{References}

[1] N. Arkani-Hamed, S. Dimopoulos and G.R. Dvali, The hierarchy problem and new dimensions at a millimeter, Phys. Lett. B 429 (1998) 263 [hep-ph/9803315] [SPIRES].

[2] G.F. Giudice, R. Rattazzi and J.D. Wells, Quantum gravity and extra dimensions at high-energy colliders, Nucl. Phys. B 544 (1999) 3 [hep-ph/9811291] [SPIRES].

[3] T. Han, J.D. Lykken and R.-J. Zhang, On Kaluza-Klein states from large extra dimensions, Phys. Rev. D 59 (1999) 105006 [hep-ph/9811350] [SPIRES].

[4] J.L. Hewett, Indirect collider signals for extra dimensions, Phys. Rev. Lett. 82 (1999) 4765 [hep-ph/9811356] [SPIRES].

[5] G.F. Giudice and A. Strumia, Constraints on extra dimensional theories from virtual graviton exchange, Nucl. Phys. B 663 (2003) 377 [hep-ph/0301232] [SPIRES].

[6] G.F. Giudice, R. Rattazzi and J.D. Wells, Transplanckian collisions at the LHC and beyond, Nucl. Phys. B 630 (2002) 293 [hep-ph/0112161] [SPIRES].

[7] LEP-II Diphoton working group, Combination of LEP-II results for the reaction $e^{+} e^{-} \rightarrow \gamma \gamma(\gamma)$ and interpretations, LEP2FF/02-02.

[8] ALEPH collaboration, Fermion pair production in $e^{+} e^{-}$collisions at high energies and limits on physics beyond the standard model, CERN-ALEPH-2001-019 (2001).

[9] DELPHI collaboration, Results on fermion-pair production at LEP running in 2000, DELPHI-2001-094-CONF-522 (2001).

[10] L3 collaboration, Search for new physics phenomena in fermion-pair production in $e^{+} e^{-}$ collisions at centre-of-mass energies up to $209 \mathrm{GeV}$, talk given at ICHEP2002, July 24-31, Amsterdam, The Netherlands (2002) [L3 NOTE 2759].

[11] OPAL collaboration, Limits on low scale quantum gravity in extra spatial dinemsions form measurements of $e^{+} e^{-} \rightarrow e^{+} e^{-}$at LEP2, OPAL Physics Note PN471.

[12] H1 collaboration, A search for contact interactions at HERA, talk given at ICHEP2002, July 24-31, Amsterdam, The Netherlands (2002).

[13] ZEUS collaboration, Search for large extra dimensions, finite quark radius and contact interactions in ep collisions at HERA, talk given at EPS2001, July 12-18, Budapest, Hungary (2001).

[14] CDF AND D0 collaboration, G.L. Landsberg, Collider searches for extra dimensions, hep-ex/0412028 [SPIRES].

[15] D0 collaboration, V.M. Abazov et al., Measurement of dijet angular distributions at $\sqrt{s}=1.96 \mathrm{Te} V$ and searches for quark compositeness and extra spatial dimensions, Phys. Rev. Lett. 103 (2009) 191803 [arXiv:0906.4819] [SPIRES].

[16] CMS collaboration, Search for large extra dimensions in dimuon events in pp collisions at $\sqrt{s}=7$ TeV, http://cdsweb.cern.ch/record/1335097/files/EXO-10-020-pas.pdf.

[17] CMS collaboration, V. Khachatryan et al., Measurement of dijet angular distributions and search for quark compositeness in pp collisions at 7 TeV, arXiv:1102.2020 [SPIRES].

[18] G. Landsberg, Quest for new physics with the first LHC data at CMS, seminar at CERN, Switzerland (2011).

[19] LEP working group, Combination of the LEP II $f^{-} f$ results, LEP2FF/02-03. 
[20] D0 collaboration, J.A. Green, Searches for compositeness at the Tevatron, hep-ex/0004035 [SPIRES].

[21] NuTeV collaboration, K.S. McFarland et al., Measurement of $\sin ^{2}\left(\Theta_{W}\right)$ from neutrino nucleon scattering at NuTeV, hep-ex/9806013 [SPIRES].

[22] S.B. Giddings and S.D. Thomas, High energy colliders as black hole factories: the end of short distance physics, Phys. Rev. D 65 (2002) 056010 [hep-ph/0106219] [SPIRES].

[23] S. Dimopoulos and G.L. Landsberg, Black holes at the LHC, Phys. Rev. Lett. 87 (2001) 161602 [hep-ph/0106295] [SPIRES].

[24] T. Banks and W. Fischler, A model for high energy scattering in quantum gravity, hep-th/9906038 [SPIRES].

[25] P. Creminelli and A. Strumia, Collider signals of brane fluctuations, Nucl. Phys. B 596 (2001) 125 [hep-ph/0007267] [SPIRES].

[26] ATLAS collaboration, G. Aad et al., Search for quark contact interactions in dijet angular distributions in pp collisions at $\sqrt{s}=7 \mathrm{TeV}$ Measured with the ATLAS detector, Phys. Lett. B 694 (2011) 327 [arXiv: 1009.5069] [SPIRES].

[27] CMS collaboration, V. Khachatryan et al., Search for dijet resonances in $7 \mathrm{TeV}$ pp collisions at CMS, Phys. Rev. Lett. 105 (2010) 211801 [arXiv:1010.0203] [SPIRES].

[28] J. Alwall et al., MadGraph/MadEvent v4: the new web generation, JHEP 09 (2007) 028 [arXiv:0706.2334] [SPIRES].

[29] N.D. Christensen et al., A comprehensive approach to new physics simulations, Eur. Phys. J. C 71 (2011) 1541 [arXiv:0906.2474] [SPIRES].

[30] CMS collaboration, S. Chatrchyan et al., Search for large extra dimensions in the diphoton final state at the Large Hadron Collider, arXiv:1103.4279 [SPIRES].

[31] I. Belotelov et al., Search for ADD extra dimensional gravity in dimuon channel with the CMS detector, CMS-NOTE-2006-076.

[32] ATLAS collaboration, G. Aad et al., Search for new physics in dijet mass and angular distributions in pp collisions at $\sqrt{s}=7 \mathrm{TeV}$ measured with the ATLAS detector, arXiv:1103.3864 [SPIRES].

[33] L. Randall and R. Sundrum, A large mass hierarchy from a small extra dimension, Phys. Rev. Lett. 83 (1999) 3370 [hep-ph/9905221] [SPIRES].

[34] G.F. Giudice, T. Plehn and A. Strumia, Graviton collider effects in one and more large extra dimensions, Nucl. Phys. B 706 (2005) 455 [hep-ph/0408320] [SPIRES].

[35] A.V. Kisselev, Interactions of cosmic neutrinos with nucleons in the RS model, Eur. Phys. J. C 42 (2005) 217 [hep-ph/0412376] [SPIRES].

[36] A.V. Kisselev, RS model with a small curvature and two-photon production at the LHC, JHEP 09 (2008) 039 [arXiv: 0804.3941] [SPIRES].

[37] T. Sjöstrand, S. Mrenna and P.Z. Skands, A brief introduction to PYTHIA 8.1, Comput. Phys. Commun. 178 (2008) 852 [arXiv:0710.3820] [SPIRES]. 